\title{
Pillar[5]arene-based [2]rotaxane: synthesis, characterization and application in coupling reaction
}

Hao Guo, ${ }^{a, b, \dagger}$ Junmei Ye, ${ }^{a, \dagger}$ Zhecheng Zhang, ${ }^{b}$ Yang Wang, ${ }^{b}$ Xiaolei Yuan, ${ }^{b}$ Changjin Ou, ${ }^{b}$ Yue Ding, ${ }^{b}$ Chaoguo Yan, *, Jin Wang, ${ }^{b}$ and Yong Yao ${ }^{*}, b$

${ }^{a}$ School of Chemistry and Chemical Engineering, Yangzhou University, Yangzhou, Jiangsu, 213300, P. R. China. ${ }^{b}$ School of Chemistry and Chemical Engineering, Nantong University, Nantong, Jiangsu, 226019, P. R. China.

Email: cgyan@yzu.edu.cn; yaoyong1986@ntu.edu.cn

\section{Supporting Information (12 pages)}

\begin{tabular}{|l|l|l|}
\hline 1. & Materials and methods & S2 \\
\hline 2. & General procedure for the preparation of guest $\boldsymbol{G}$ & S3 \\
\hline 3. & General procedure for the preparation of [2] rotaxane $\boldsymbol{R}$ & S5 \\
\hline 4. & Catalytic activities & S9 \\
\hline
\end{tabular}




\section{Materials and methods}

All reagents were commercially available and used as supplied without further purification. Deuterated solvents were purchased from Cambridge Isotope Laboratory (Andover, MA). NMR spectra were recorded on a Varian Unity $400 \mathrm{MHz}$ spectrometer. Mass spectra were recorded on a Micromass Quattro II triple-quadrupole mass spectrometer using electrospray ionization with a MassLynx operating system. These samples were prepared by drop-coating the three solutions onto a carbon-coated copper grid. TEM experiments were performed on a JEM$1200 \mathrm{EX}$ instrument with an accelerating voltage of $80 \mathrm{kV}$. ICP-EOS was performed on PerkinElmer-NexION 350. The density functional theory (DFT) calculation was performed using the $\mathrm{Dmol}^{3}$ code $^{[1]}$. The exchange-correlation interaction was treated by the generalized gradient approximation (GGA) with PBE functional ${ }^{[2]}$. A double numerical quality basis set with d-type polarization function $\left(\mathrm{DNP}^{[3]}\right)$ was utilized for all the geometric optimizations, total energy calculations. The core electrons were modeled using effective core pseudopotentials (ECP) by Dolg ${ }^{[4]}$ and Bergner ${ }^{[5]}$. All calculations were spin unrestricted. The positions of all the atoms were fully relaxed until the following convergence criterion are met respectively: $0.002 \mathrm{Ha} / \AA$ for force, $10^{-5} \mathrm{Ha}$ for total

energy and $0.005 \AA$ for displacement. The real space cutoff radius was $4.1 \AA$. The self-consistent field computations criterion was chosen to be $10^{-6} \mathrm{Ha}$.

Surface area measurements were conducted on a BELSORP-Max Accelerated Surface Area and Porosimetry Analyzer. The sample $(91.3 \mathrm{mg})$ was degassed at $100{ }^{\circ} \mathrm{C}$ for $12.0 \mathrm{~h}$ and then backfilled with $\mathrm{N} 2$. N2 isotherms were generated by incremental exposure to ultra high purity nitrogen up to $1.0 \mathrm{~atm}$ in a liquid nitrogen bath $(77.0 \mathrm{~K})$, and surface parameters were determined using BET adsorption models included in the instrument software (BELSORPMax). 


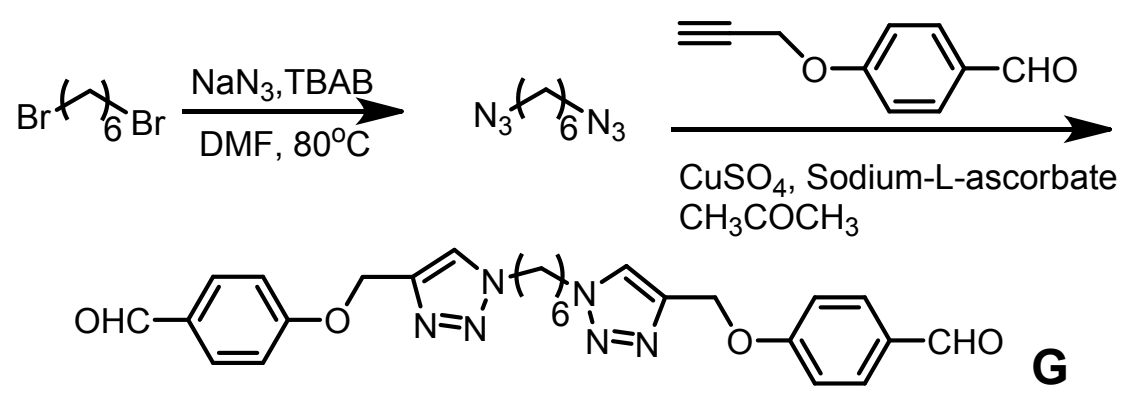

Scheme S1. Synthetic route to guests $\mathbf{G}$.

Synthesis of G:

In a $100 \mathrm{~mL}$ round bottom flask, 1, 6-dibromohexane (2.41 g, $10 \mathrm{mmol}_{\text {.), }} \mathrm{NaN}_{3}(1.625 \mathrm{~g}, 25 \mathrm{mmol}$ ), TBAB (0.166g, 0.5 mmol) and DMF $(20 \mathrm{~mL})$ were added. The reaction mixture was stirred at $80^{\circ} \mathrm{C}$ for 3 hours. After the vessel was cooled back to room temperature, the mixture was added in $\mathrm{CH}_{2} \mathrm{Cl}_{2}(50 \mathrm{~mL})$ and $\mathrm{H}_{2} \mathrm{O}(50 \mathrm{~mL})$, and washed with $\mathrm{H}_{2} \mathrm{O}(2 \times$ $100 \mathrm{~mL}$ ). The organic phase was dried over anhydrous $\mathrm{Na}_{2} \mathrm{SO}_{4}$, filtered and concentrated in vacuo to give intermediate 1 , 6-diazidohexane. Sodium-L-ascorbate $(0.099 \mathrm{~g}, 0.5 \mathrm{mmol})$ was added to a mixture of 1, 6-diazidohexane $(1.79 \mathrm{~g}, 5$ mmol), 4-(prop-2-yn-1-yloxy)benzaldehyde $(1.600 \mathrm{~g}, 0.5 \mathrm{mmol})$ and a solution of $\mathrm{CuSO}_{4} \cdot 5 \mathrm{H}_{2} \mathrm{O}(20 \mathrm{mg}, 5 \mathrm{~mL}$ in methanol) in acetone $(25 \mathrm{~mL})$. The mixture was stirred at $50^{\circ} \mathrm{C}$ for $12 \mathrm{~h}$. After completion, the cooled reaction mixture was concentrated under vacuum and added to $\mathrm{CH}_{2} \mathrm{Cl}_{2}(25 \mathrm{~mL})$. The solution was washed with solution that had been saturated with $\mathrm{NH}_{4} \mathrm{Cl}(2 \times 25 \mathrm{~mL})$ and $\mathrm{H}_{2} \mathrm{O}(2 \times 25 \mathrm{~mL})$. The organic phase was dried over anhydrous $\mathrm{Na}_{2} \mathrm{SO}_{4}$, and concentrated in vacuo. The guest $\mathbf{G}$ was obtained by recrystallization with ethanol.

G: White solid, 53\%, m.p. $118-120^{\circ} \mathrm{C} ;{ }^{1} \mathrm{H}$ NMR $\left(400 \mathrm{MHz}, \mathrm{CDCl}_{3}\right) \delta: 9.88$ (s, 2H, CHO), 7.84 (d, J=8.8 Hz, 4H, ArH), 7.66 (s, 2H, ArH), 7.11 (d, $J=8.8 \mathrm{~Hz}, 4 \mathrm{H}, \mathrm{ArH}), 5.30$ (s, 4H, OCH$), 4.35$ (t, $J=7.0 \mathrm{~Hz}, 4 \mathrm{H}, \mathrm{OCH}_{2}$ ), 1.91 (brs, 4H, $\mathrm{CH}_{2}$ ), 1.35 (brs, 4H, $\mathrm{CH}_{2}$ ); ${ }^{13} \mathrm{C}$ NMR (100 MHz, $\mathrm{CDCl}_{3}$ ) $\delta: 234.3,190.7,163.1,131.9,130.1,115.0,62.0,50.1,29.8$, 25.6; IR (KBr) v: 3130, 2928, 2858, 2826, 2743, 1687, 1602, 1573, 1505, 1469, 1425, 1389, 1302, 1247, 1158, 1111 , 1049, 994, 868, 837, 777, 733, 689, $639 \mathrm{~cm}^{-1}$; MS (m/z): HRMS (ESI) Calcd. for $\mathrm{C}_{26} \mathrm{H}_{28} \mathrm{~N}_{6} \mathrm{NaO}_{4}\left([\mathrm{M}+\mathrm{Na}]^{+}\right): 511.2070$, found: 511.2076 . 


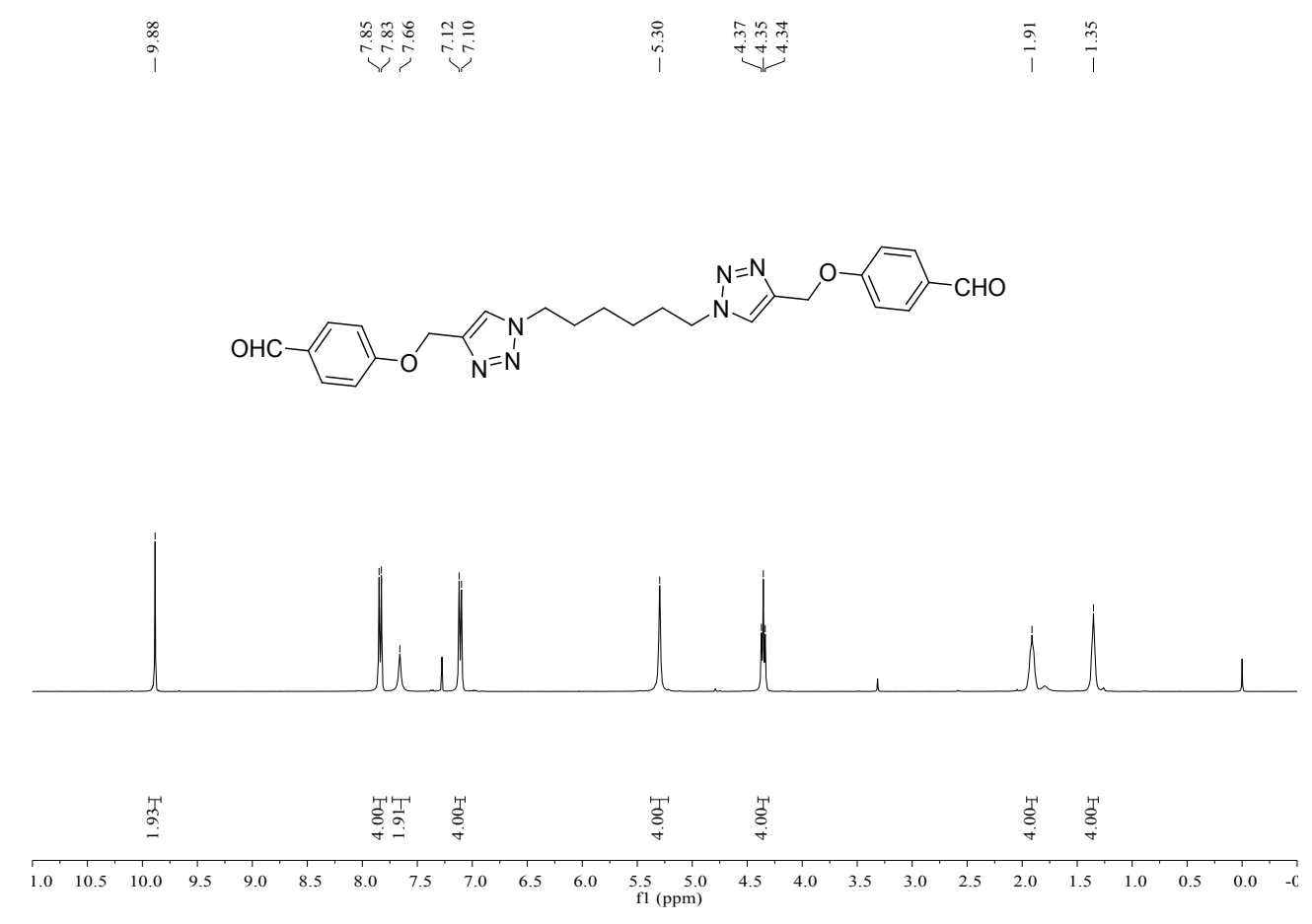

Figure S1 ${ }^{1} \mathrm{H}$ NMR spectrum (400 MHz, $\left.\mathrm{CDCl}_{3}, 293 \mathrm{~K}\right)$ of $\mathbf{G}$.

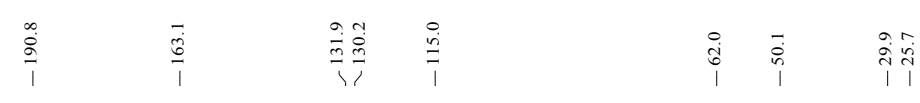

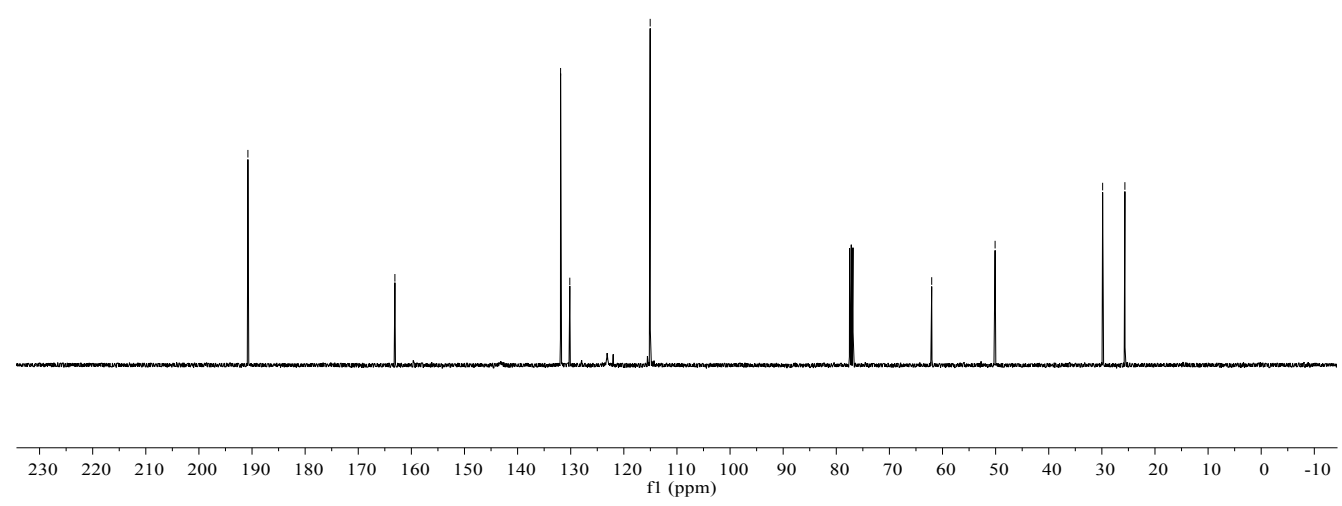

Figure $\boldsymbol{S} 2{ }^{13} \mathrm{C}$ NMR spectrum $\left(100 \mathrm{MHz}, \mathrm{CDCl}_{3}, 293 \mathrm{~K}\right)$ of $\mathbf{G}$. 


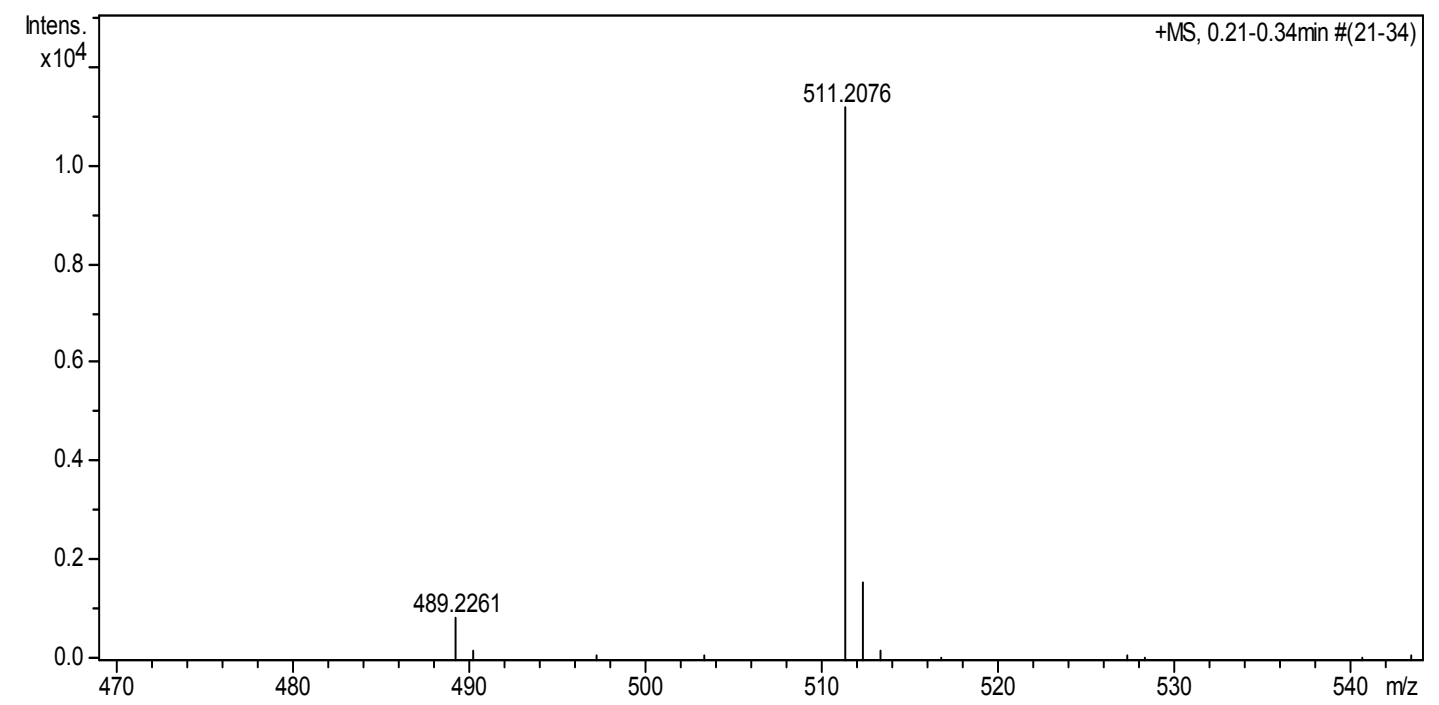

Figure S3 Mass spectra of $\mathbf{G}$ Calcd. for $\mathrm{C}_{26} \mathrm{H}_{28} \mathrm{~N}_{6} \mathrm{NaO}_{4}\left([\mathrm{M}+\mathrm{Na}]^{+}\right)$: 511.2076.

3. General procedure for the preparation of [2] rotaxanes $R$
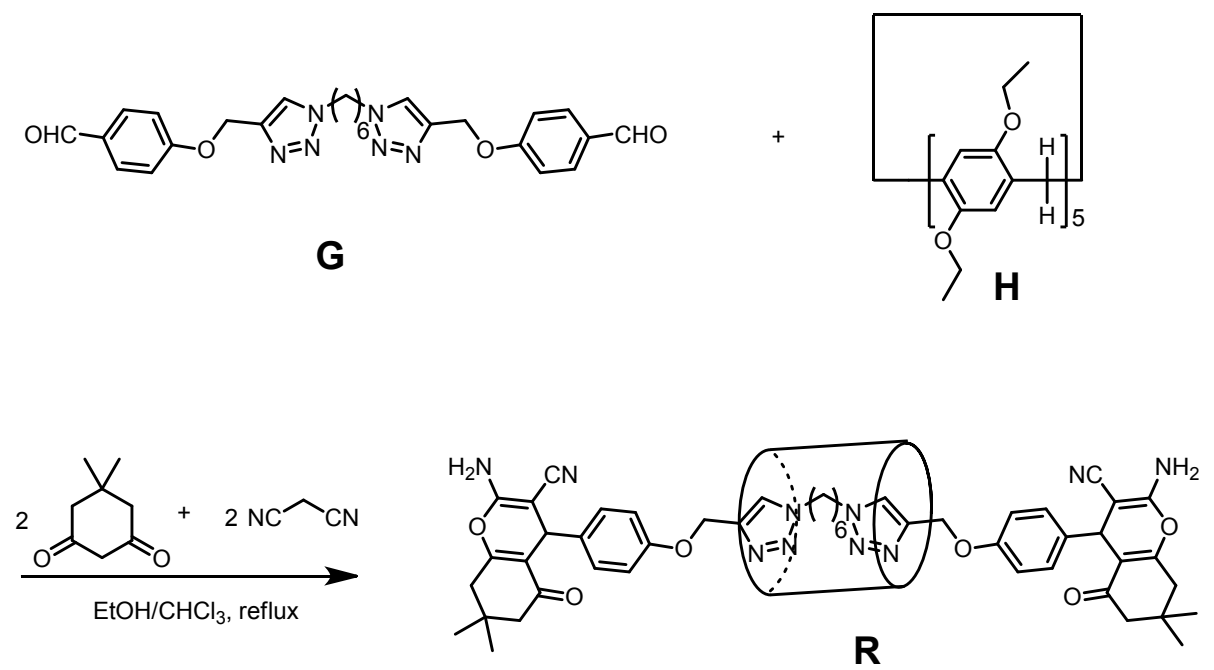

Scheme S2. Synthetic route to [2]rotaxanes $R$.

\section{Synthesis of R:}

In a $50 \mathrm{~mL}$ round bottom flask, $\mathbf{G}(0.51 \mathrm{~g}, 10 \mathrm{mmol}$ ), pillar[5]arene (0.890 g, $1 \mathrm{mmol}$.), 5,5dimethylcyclohexane-1,3-dione $(0.140 \mathrm{~g}, 1 \mathrm{mmol})$, malononitrile $(0.066 \mathrm{~g}, 1 \mathrm{mmol})$, and piperidine $(2$ drops) were added in a mixed solvent (EtOH: $\left.\mathrm{CHCl}_{3}=1: 1\right)$. The reaction mixture was refluxed for 3 hours. After completion, the cooled reaction mixture was concentrated under vacuum and subjected to silica column chromatography using a mixture of DCM and $\mathrm{MeOH}(v / v=25: 1)$ as eluent to give crude product. The pure product $\mathbf{R}$ was obtained by recrystallization with ethanol. 
R: Yellow solid, 25\%, m.p. 147-150 ${ }^{\circ}$; ${ }^{1} \mathrm{H}$ NMR $\left(400 \mathrm{MHz}, \mathrm{DMSO}-d_{6}\right) \delta: 7.66$ (s, 2H, ArH), $7.11(\mathrm{~d}, J=7.8 \mathrm{~Hz}, 4 \mathrm{H}$, ArH), 6.99 (d, $\left.J=8.4 \mathrm{~Hz}, 4 \mathrm{H}, \mathrm{NH}_{2}\right), 6.97$ (s, 4H, ArH), $6.86(\mathrm{~s}, 10 \mathrm{H}, \mathrm{ArH}), 5.20$ (s, 4H, OCH $), 4.16$ (s, 2H, CH), 3.79$3.77\left(\mathrm{~m}, 20 \mathrm{H}, \mathrm{OCH}_{2}\right), 3.64$ (s, $\left.10 \mathrm{H}, \mathrm{CH}_{2}\right), 3.10$ (brs, 4H, $\left.\mathrm{CH}_{2}\right), 2.53$ (d, $\left.J=17.4 \mathrm{~Hz}, 2 \mathrm{H}, \mathrm{CH}_{2}\right), 2.45(\mathrm{~d}, J=18.0 \mathrm{~Hz}, 2 \mathrm{H}$, $\mathrm{CH}_{2}$ ), 2.27-2.23 (m, 2H, CH (d, $J=6.6 \mathrm{~Hz}, 6 \mathrm{H}, \mathrm{CH}_{3}$ ), -0.03 (brs, $4 \mathrm{H}, \mathrm{CH}_{2}$ ), -1.95 (brs, 4H, $\mathrm{CH}_{2}$ ); ${ }^{13} \mathrm{C}$ NMR (100 MHz, DMSO- $d_{6}$ ) $\delta: 195.9,162.5$, 162.4, 158.9, 157.2, 157.2, 149.5, 143.3, 137.6, 137.6, 128.7, 128.3, 122.3, 120.1, 114.7, 114.4, 113.4, 63.8, 61.7, 61.6, $58.8,58.8,50.4,49.3,35.2,32.1,29.5,29.2,28.9,28.7,27.2,27.1,23.0,15.4$; IR (KBr) v: 3174, 2975, 2935, 2873, 2735 , 2195, 1682, 1608, 1500, 1478, 1441, 1406, 1391, 1364, 1302, 1249, 1210, 1176, 1159, 1111, 1052, 957, 899, 880, 860, 843, 779, 731, 714, 659, $628 \mathrm{~cm}^{-1}$; MS (m/z): HRMS (ESI) Calcd. for $\mathrm{C}_{103} \mathrm{H}_{123} \mathrm{~N}_{10} \mathrm{O}_{16}\left([\mathrm{M}+\mathrm{H}]^{+}\right)$: 1756.9152, found: 1756.9103.
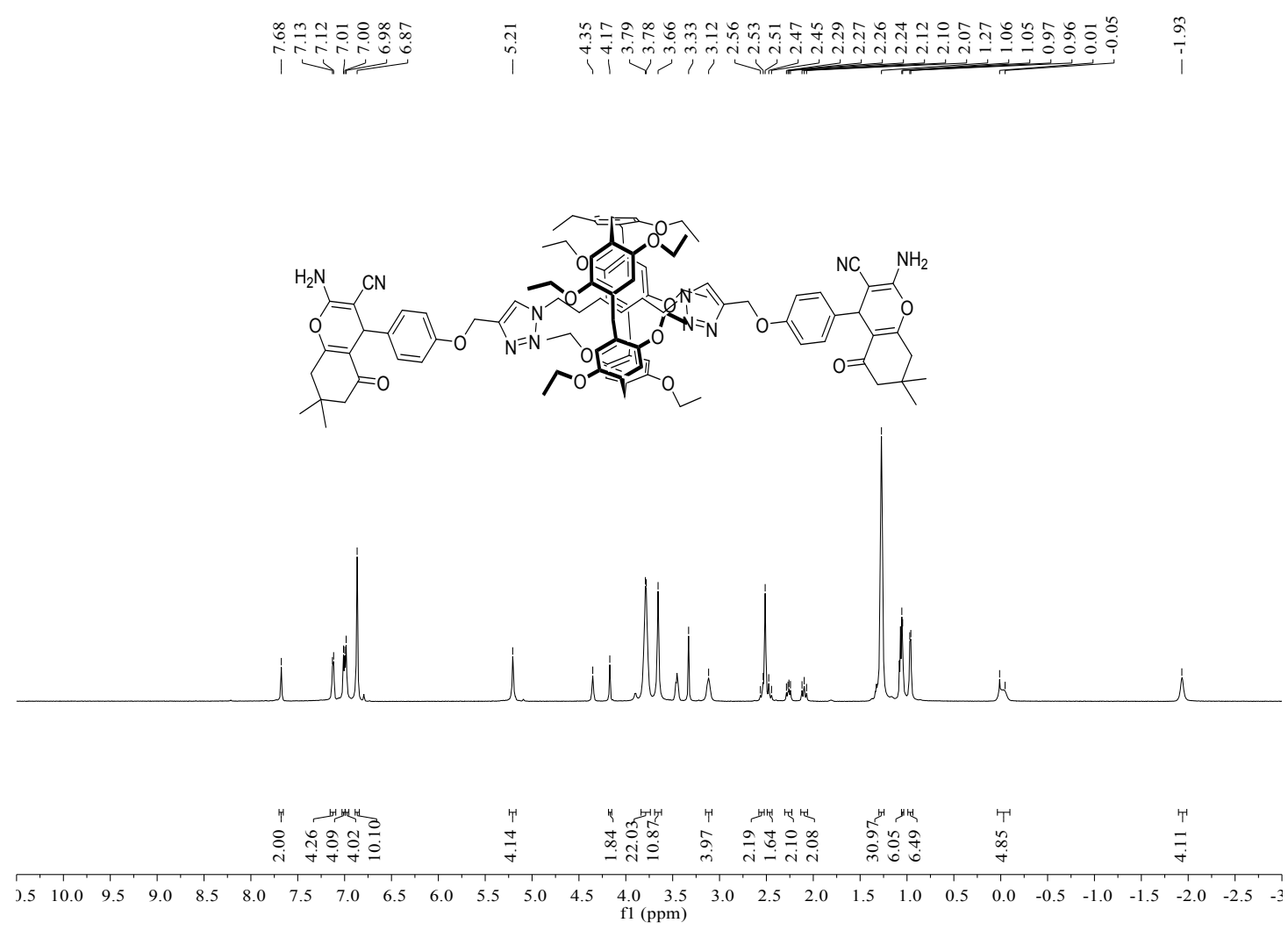

Figure $\boldsymbol{S} 4{ }^{1} \mathrm{H}$ NMR spectrum (400 MHz, DMSO- $d_{6}, 293 \mathrm{~K}$ ) of $\mathbf{R}$. 


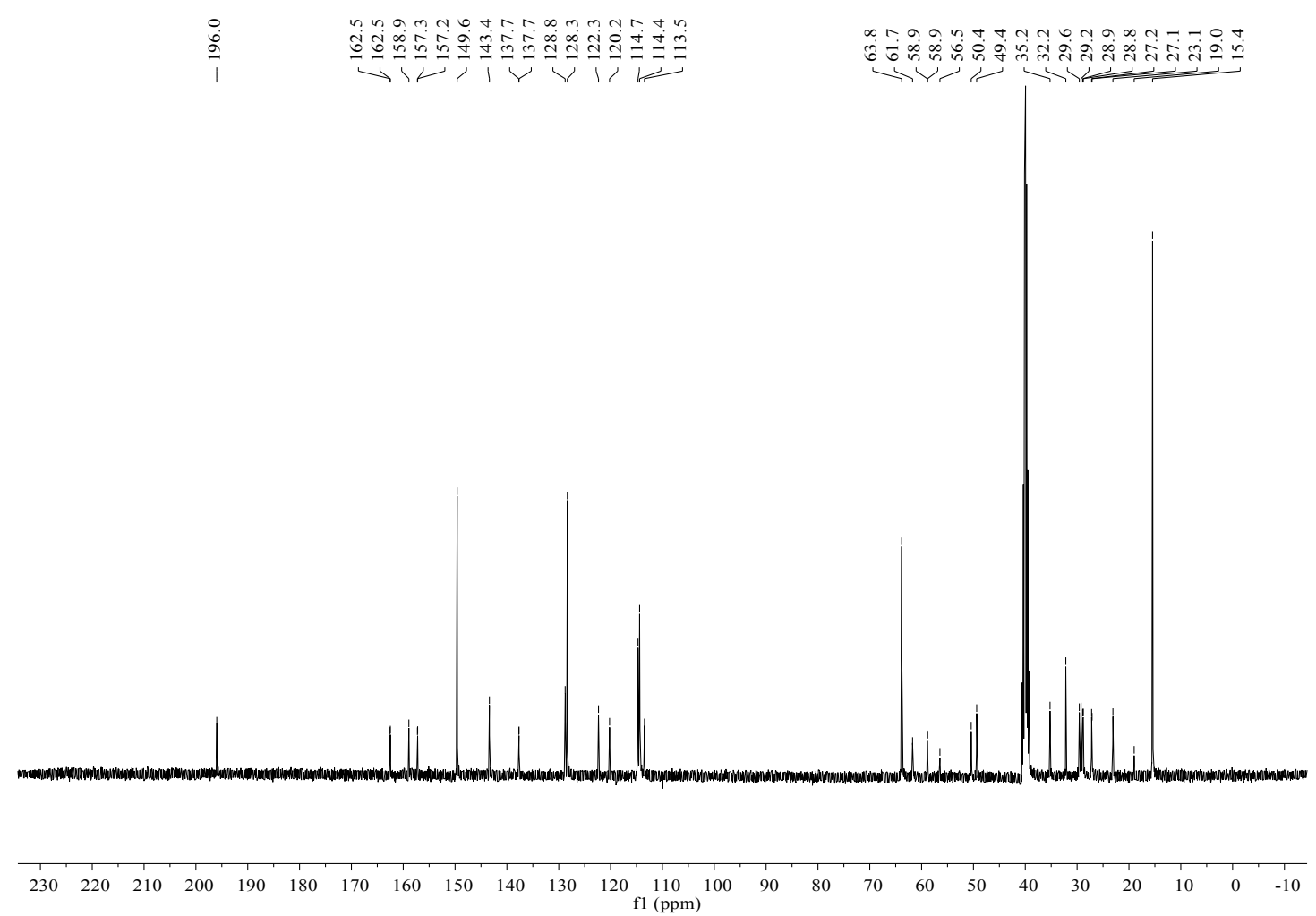

Figure $S 5{ }^{13} \mathrm{C}$ NMR spectrum (100 MHz, DMSO- $\left.d_{6}, 293 \mathrm{~K}\right)$ of $\mathbf{R}$.

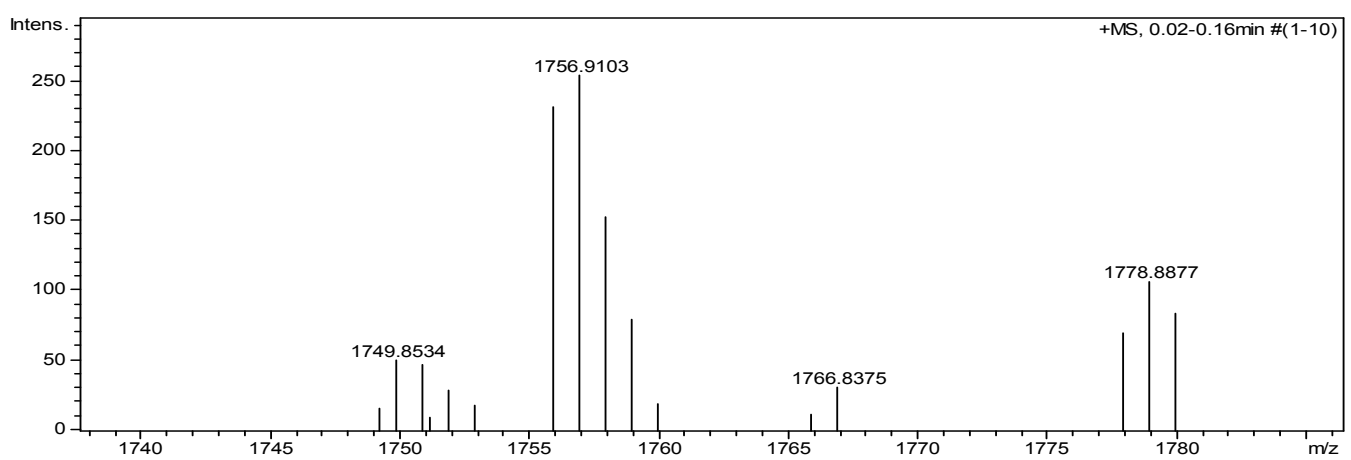

Figure S6 Mass spectra of $\mathbf{R}$ Calcd. for $\mathrm{C}_{103} \mathrm{H}_{123} \mathrm{~N}_{10} \mathrm{O}_{16}\left([\mathrm{M}+\mathrm{H}]^{+}\right)$: 1756.9152, found: 1756.9103 .

1: ${ }^{1} \mathrm{H}$ NMR (400 MHz, DMSO- $\left.d_{6}\right) \delta: 8.21(\mathrm{~s}, 2 \mathrm{H}, \mathrm{ArH}), 7.06(\mathrm{~d}, J=8.8 \mathrm{~Hz}, 4 \mathrm{H}, \mathrm{ArH}), 6.97\left(\mathrm{~m}, 8 \mathrm{H}, \mathrm{ArH}, \mathrm{NH}_{2}\right), 5.08(\mathrm{~s}$, $\left.4 \mathrm{H}, \mathrm{CH}_{2}\right), 4.34\left(\mathrm{t}, J=7.0 \mathrm{~Hz}, 4 \mathrm{H}, \mathrm{CH}_{2}\right), 4.12(\mathrm{~s}, 2 \mathrm{H}, \mathrm{CH}), 2.55-2.50\left(\mathrm{~m}, 2 \mathrm{H}, \mathrm{CH}_{2}\right), 2.50-2.45\left(\mathrm{~m}, 2 \mathrm{H}, \mathrm{CH}_{2}\right), 2.24(\mathrm{~d}, J=$ $16.0 \mathrm{~Hz}, 2 \mathrm{H}, \mathrm{CH}_{2}$ ), 2.09 (d, J=16.0 Hz, 2H, $\mathrm{CH}_{2}$ ), 1.80 (brs, 4H, $\mathrm{CH}_{2}$ ), 1.26 (brs, 4H, $\mathrm{CH}_{2}$ ), 1.03 (s, 6H, $\mathrm{CH}_{3}$ ), 0.94 (s, $\left.6 \mathrm{H}, \mathrm{CH}_{3}\right) .{ }^{13} \mathrm{C} \mathrm{NMR}\left(100 \mathrm{MHz}, \mathrm{CDCl}_{3}\right) \delta: 196.1,161.5,157.6,157.6,157.2,144.2,136.3,136.2,128.7,122.6,118.9$, $118.9,115.4,114.8,114.0,77.3,77.0,76.7,63.1,63.0,62.0,50.7,50.1,40.6,34.8,32.2,29.9,28.8,27.7,25.7$ 


\section{$\vec{a}$}
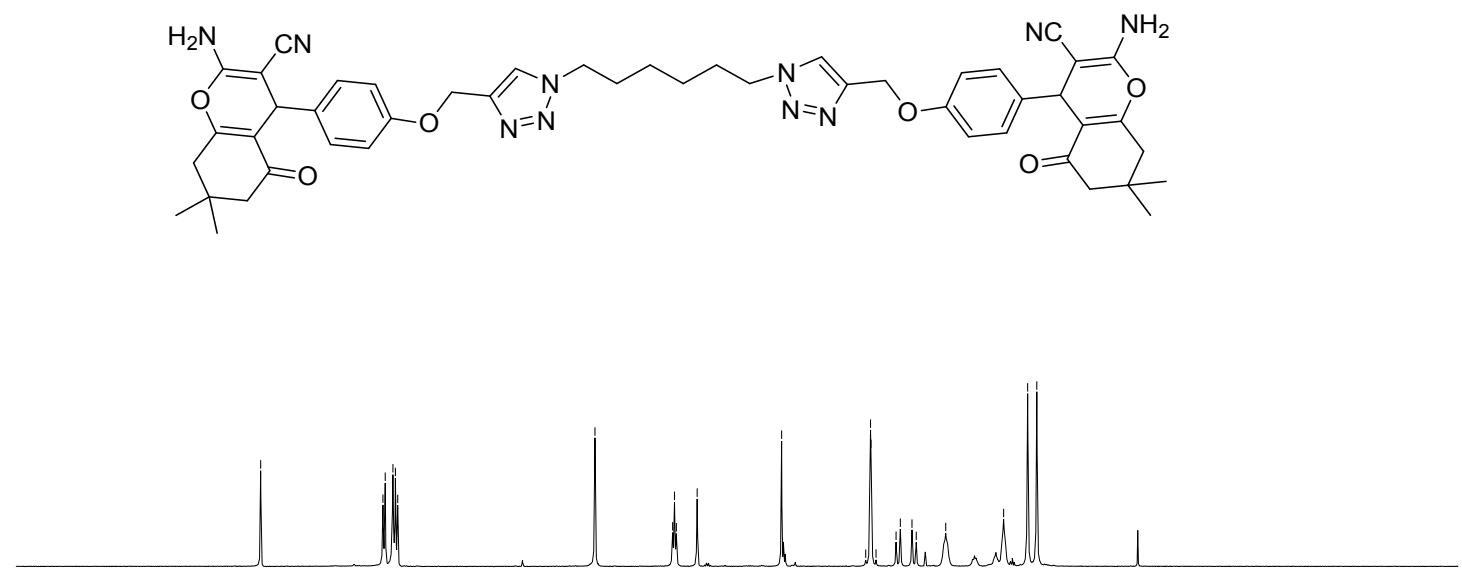

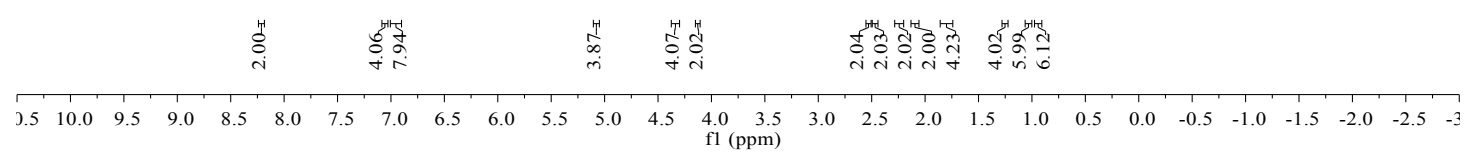

Figure $S 7^{1} \mathrm{H}$ NMR spectrum (400 MHz, DMSO- $d_{6}, 293 \mathrm{~K}$ ) of 1 .

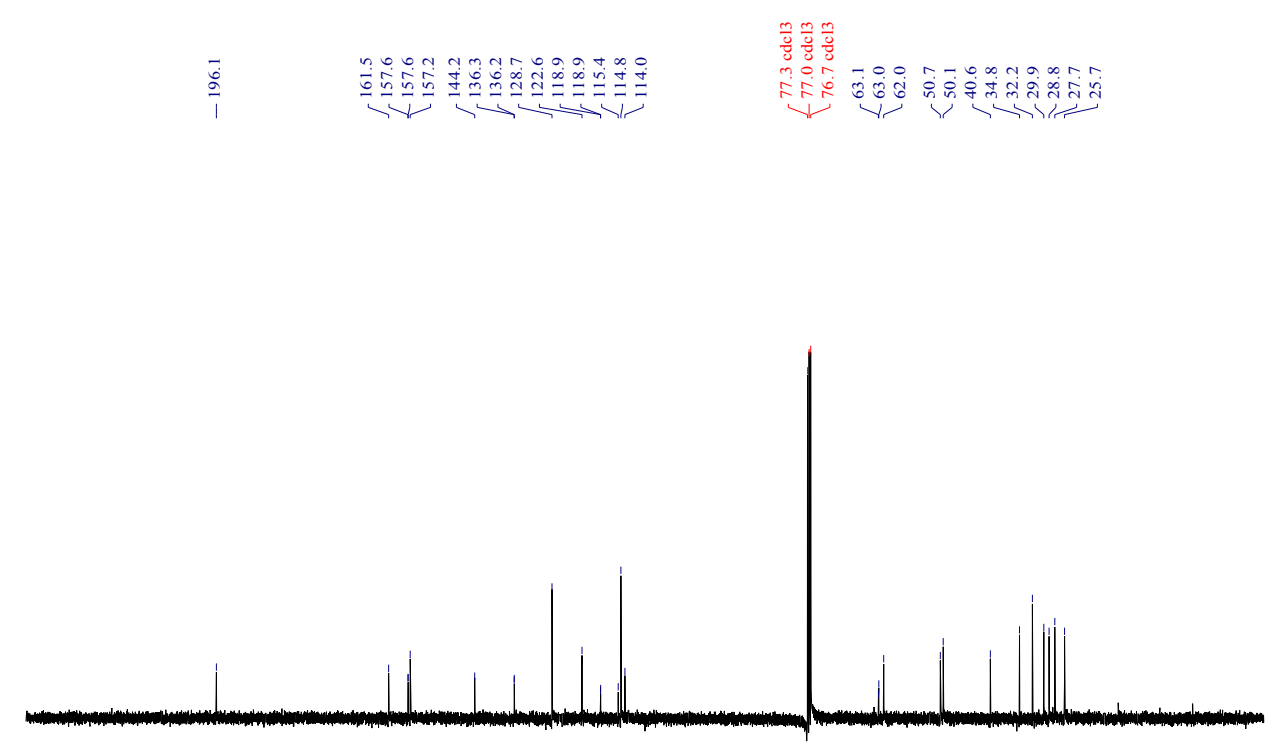

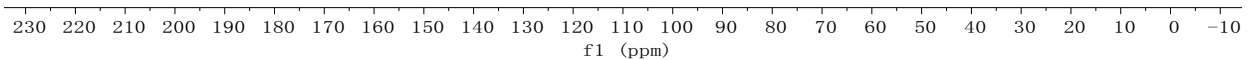

Figure $\boldsymbol{S} \boldsymbol{8}^{13} \mathrm{C}$ NMR spectrum $\left(100 \mathrm{MHz}, \mathrm{CDCl}_{3}, 293 \mathrm{~K}\right)$ of $\mathbf{1}$. 


\section{Catalytic activities}

Construction of R@Pd catalyst. R $(175 \mathrm{mmg}, 1.00 \mathrm{mmol})$ was dissolved in $2 \mathrm{~mL}$ DMF, Pd( $\left(\mathrm{NO}_{3}\right)_{2}(23 \mathrm{mg}, 1.00 \mathrm{mmol})$ was added in to the $\mathbf{R}^{\prime}$ ' solution, the mixture was stirred for $1 \mathrm{~h}$ at r.t. Then the precipitation was collected and washed with $\mathrm{CH}_{2} \mathrm{Cl}_{2}$ twice (160 mg, yield: $81 \%$ ).

The percent incorporation of palladium in the materials was about $5.1 \%$ tested by ICP-EOS.

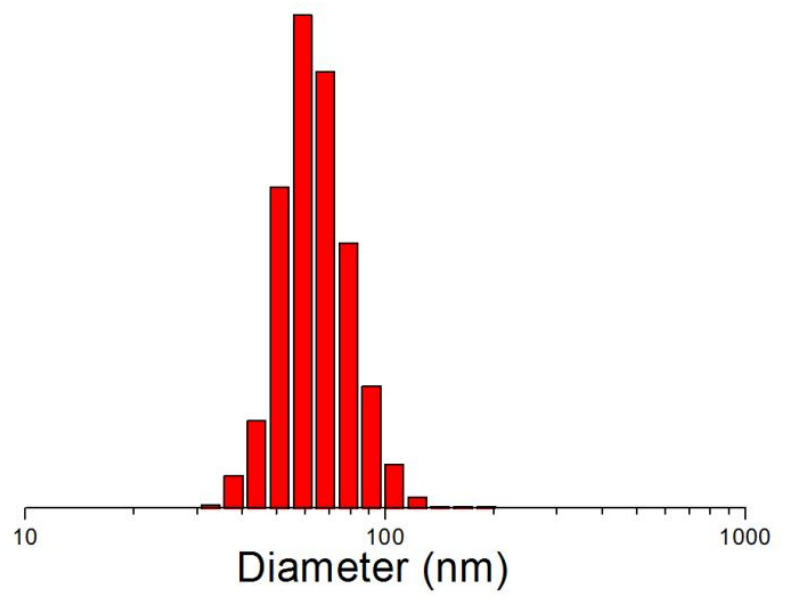

Figure $\mathbf{S} 9$ DLS study of $\operatorname{Pd} @ \mathbf{R}$ dispersed in water.

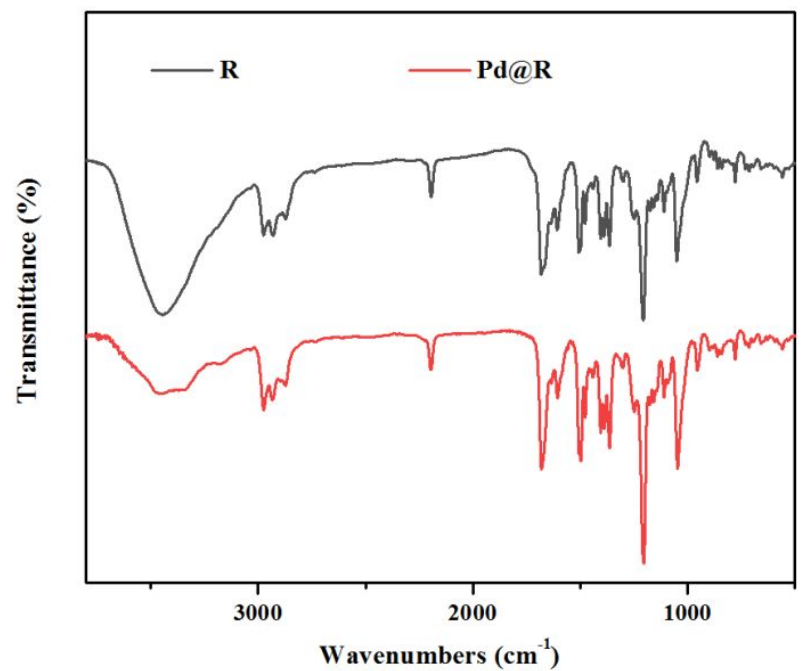

Figure S10 FI-IR spectra of pillar[5]arene-based [2]rotaxane $\mathbf{R}$ and the obtained catalyst Pd@R. 


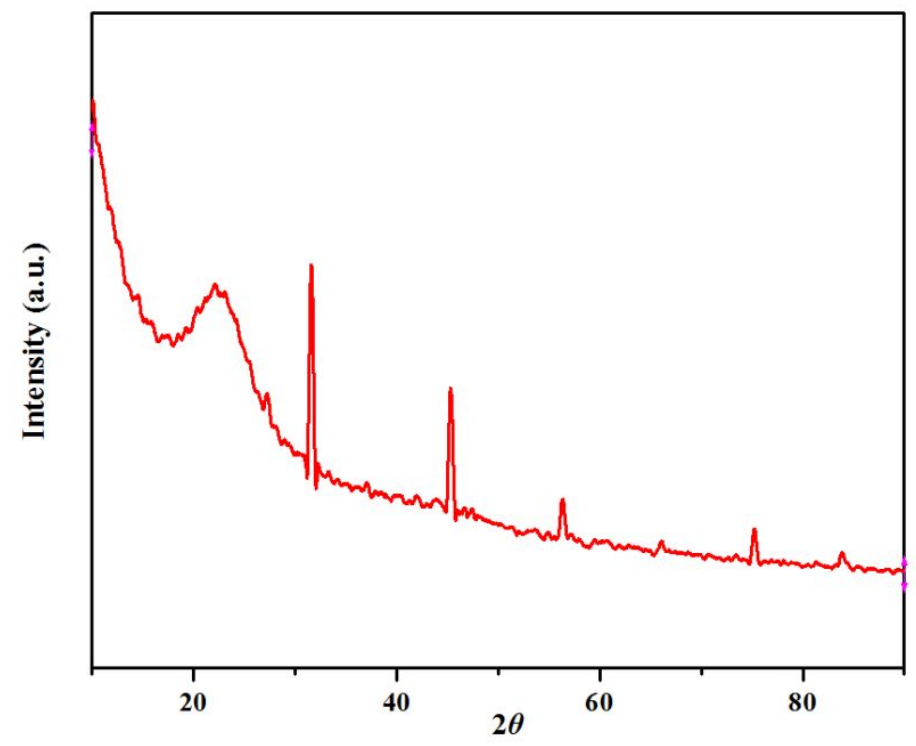

Figure S11 XRD pattern of Pd@R catalyst.

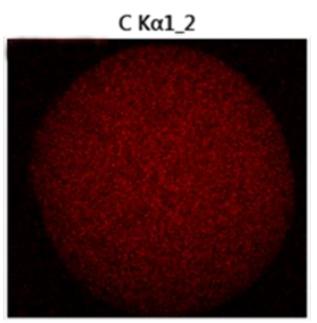

Pd L $\alpha 1$

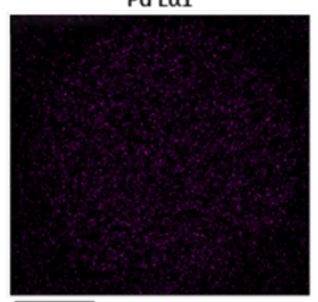

Figure S12. SEM mapping of Pd@R catalyst.

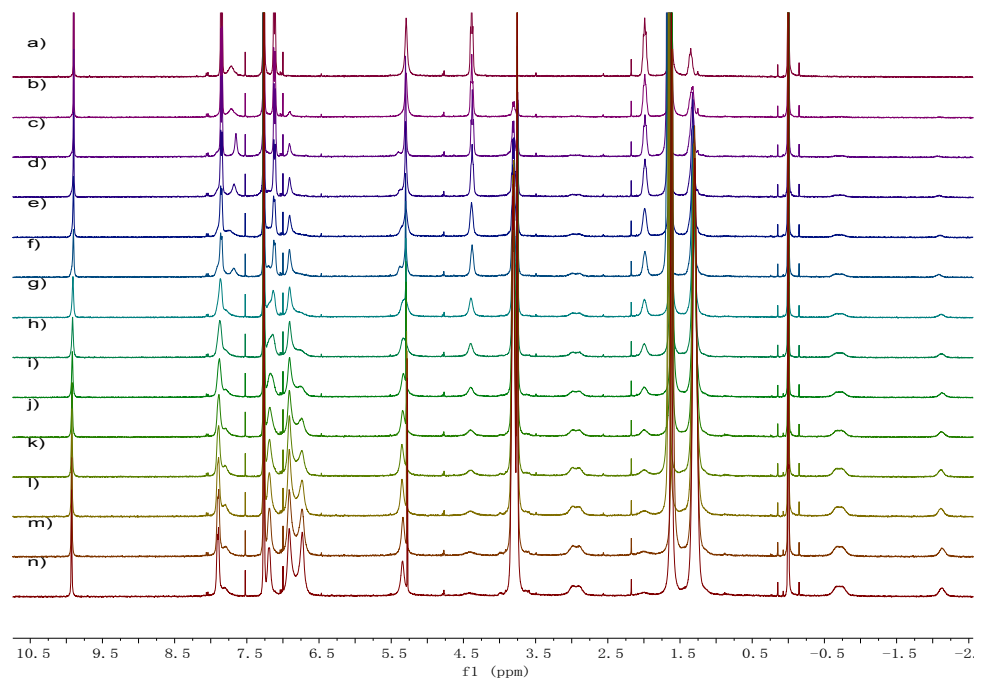

Figure S13 ${ }^{1} \mathrm{H}$ NMR spectra $\left(400 \mathrm{MHz}, \mathrm{CDCl}_{3}, 298 \mathrm{~K}\right)$ of $\mathbf{G}$ at a constant concentration of $3.0 \mathrm{mM}$ with different concentrations of P5 (mM): (a) 0.0, (b) 0.2, (c) 0.4, (d) 0.6, (e) 0.8, (f) 1.0, (g) 1.4, (h) 1.8, (i) 2.2, (j) 2.6, (k) 3.0, (l) 3.4, (m) 3.8 and (n) 4.2 . 

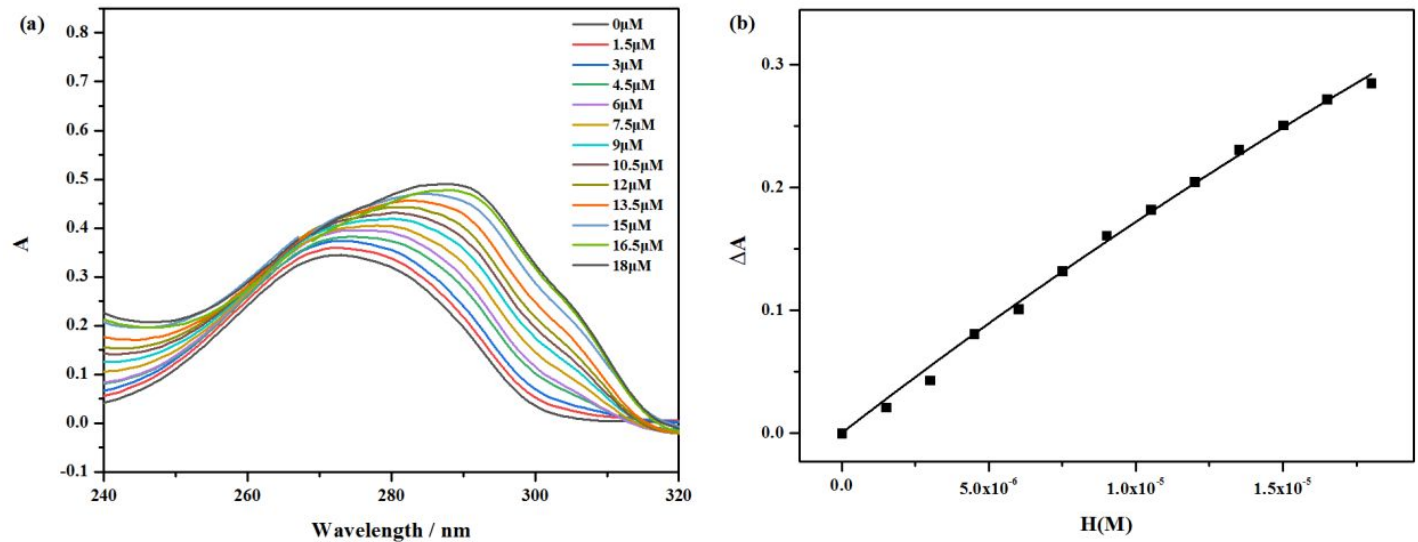

Figure S14 a) UV-Vis absorption spectra of $\mathbf{G}$ at a constant concentration of $15.0 \mu \mathrm{M}$ with different concentrations of P5 $(\mu \mathrm{M})$. b) The absorption changes of $\mathbf{G}$ upon the addition of $\mathbf{P 5}$. The black solid line was obtained from the non-linear curvefitting using eq. S1. The association constant of $\mathbf{W P 5}$ and $\mathbf{G}$ was estimated to be $(1.04 \pm 0.46) \times 10^{4} \mathrm{M}^{-1}$.

$\Delta \delta=\left(\Delta \delta \infty /[\mathrm{G}]_{0}\right)\left(0.5[\mathrm{H}]_{0}+0.5\left([\mathrm{G}]_{0}+1 / K_{a}\right)-\left(0.5\left([\mathrm{H}]_{0}^{2}+\left(2[\mathrm{H}]_{0}\left(1 / K_{a}-[\mathrm{G}]_{0}\right)\right)+\left(1 / K_{a}+[\mathrm{G}]_{0}\right)^{2}\right)^{0.5}\right)\right)$ (eq.S1)

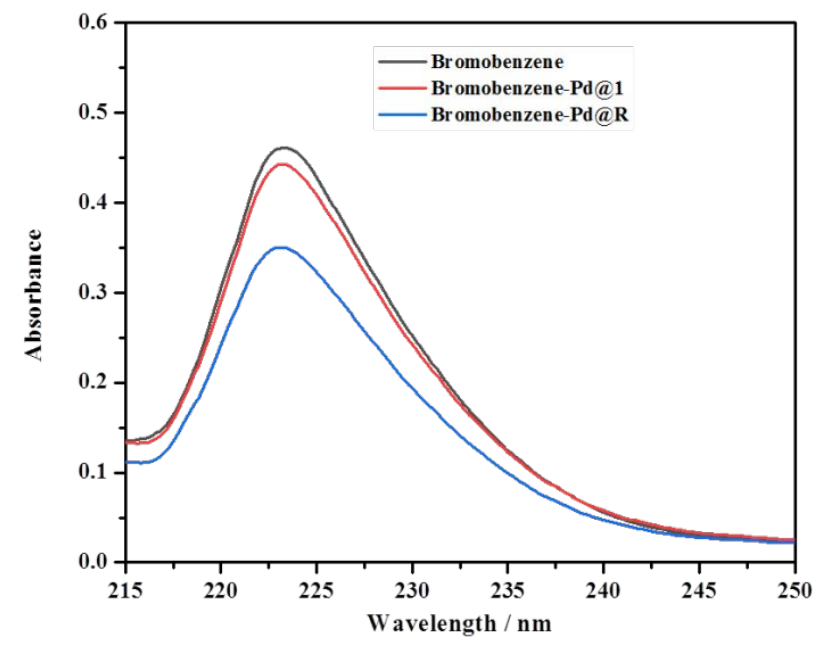

Figure S15 The UV-vis absorbance of Bromobenzene $(10 \mu \mathrm{g} / \mathrm{mL})$, Bromobenzene-Pd@1 ([Bromobenzene] $=10 \mu \mathrm{g} / \mathrm{mL}$, $[\mathbf{P d} @ 1]=30 \mu \mathrm{g} / \mathrm{mL})$, Bromobenzene-Pd@R ([Bromobenzene $]=10 \mu \mathrm{g} / \mathrm{mL},[\mathbf{P d} @ \mathbf{R}]=30 \mu \mathrm{g} / \mathrm{mL})$ in $\mathrm{CH}_{2} \mathrm{Cl}_{2}$. 


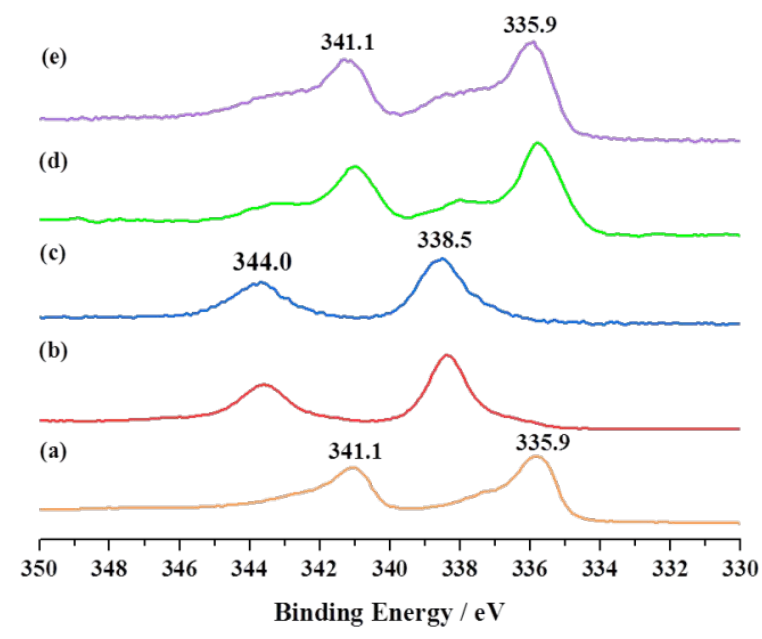

Figure S16 XPS analysis for Pd 3d spectra: (a) Pd/C; (b) Pd(NO $)_{2}$; (c) Pd@R; (d) Pd@R recycled after one time of the Suzuki-Miyaura coupling reaction; (e) $\mathrm{Pd} @ \mathbf{R}$ recycled after three times of the Suzuki-Miyaura coupling reaction.

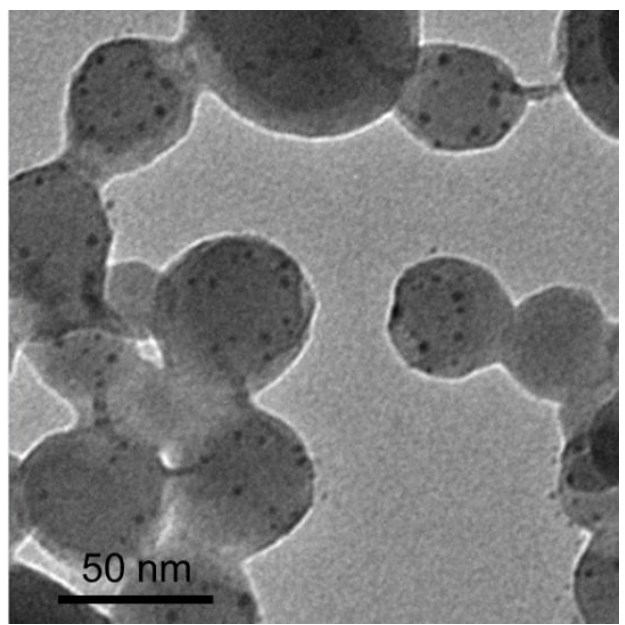

Figure S17 TEM image of Pd@R recycled after three times of the Suzuki-Miyaura coupling reaction

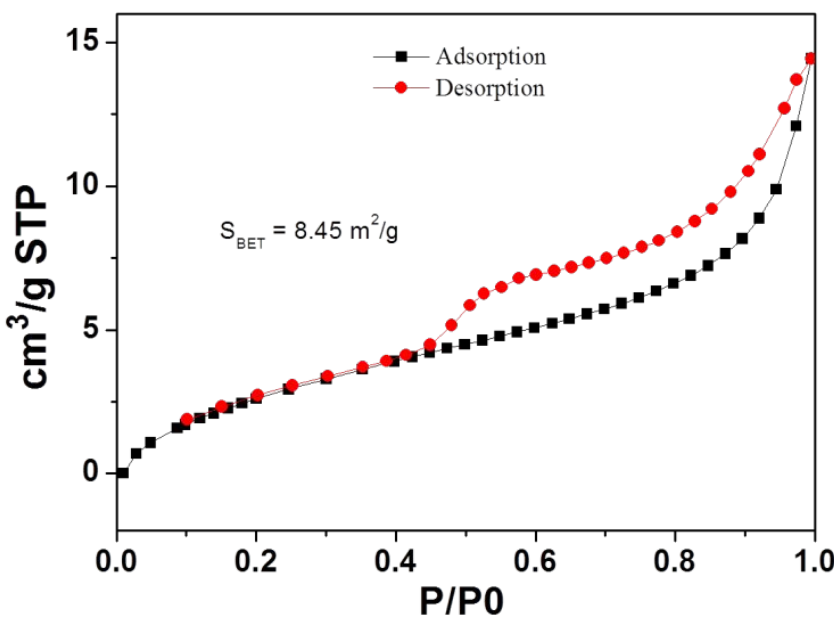

Figure $\mathbf{S 1 8} \mathrm{N}_{2}$ sorption isotherm $(77.0 \mathrm{~K})$ and surface area data analysis of polymer Pd@R. 


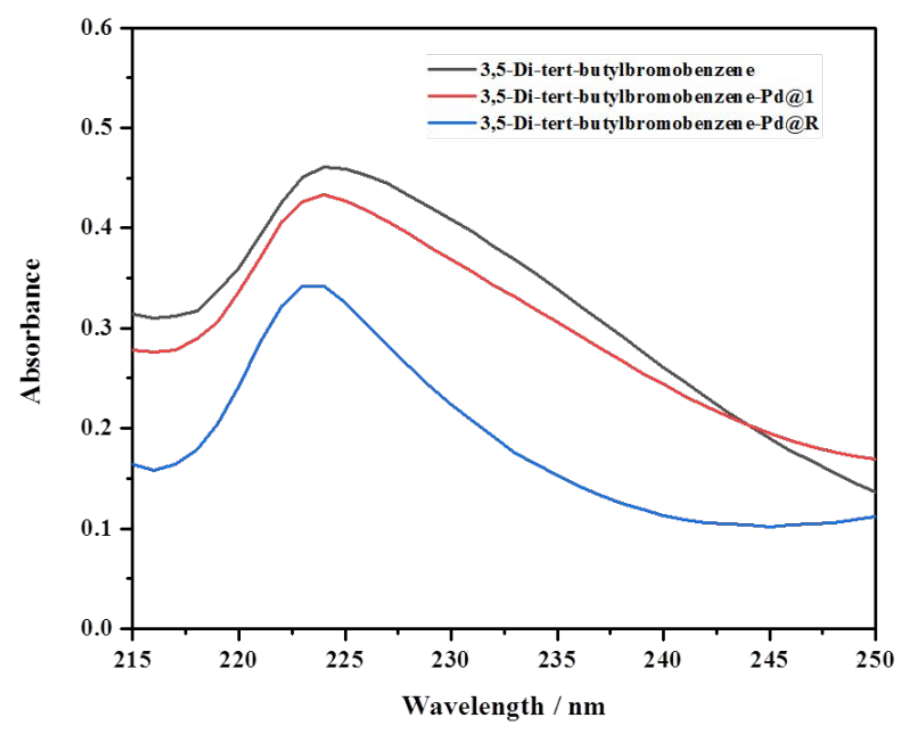

Figure S19 The UV-vis absorbance of 3,5-Di-tert-butylBromobenzene (10 $\mu \mathrm{g} / \mathrm{mL})$, 3,5-Di-tert-butylBromobenzene-

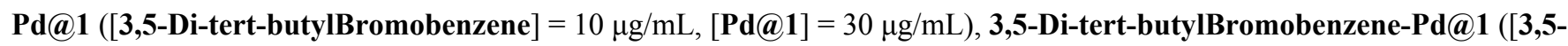

Di-tert-butylBromobenzene $]=10 \mu \mathrm{g} / \mathrm{mL},[\mathbf{P d} @ \mathbf{R}]=30 \mu \mathrm{g} / \mathrm{mL})$ in $\mathrm{CH}_{2} \mathrm{Cl}_{2}$.

General Procedure for the Suzuki-Miyaura Coupling Reaction: In a typical process, phenyl-OTf (1.00 mmol), 4boronic acid pyridine $(1.50 \mathrm{mmol}), \mathrm{K}_{2} \mathrm{CO}_{3}(2.00 \mathrm{mmol})$, catalyst $(0.50 \mathrm{~mol} \% \mathrm{Pd})$, DMF $(5 \mathrm{~mL}), 100{ }^{\circ} \mathrm{C}$. Yields were determined by GC/MS (Agilent 1260). We also used $\mathrm{H}_{2} \mathrm{O}$ to instead DMF, the conversation was about $70 \%$ under the same condition. So in this article we choose DMF as the solvent.

The recycle process: After the end of the reaction, $\mathrm{Pd} @ \mathbf{R}$ was collected by filtration and then washed with water and DMF twice, dried in vacuo, and then employed for the next run.

Table S1. Suzuki-Miyaura coupling reaction catalyzed by Pd catalysts

\begin{tabular}{|c|c|c|c|}
\hline \multicolumn{3}{|c|}{ Yields of conversion (\%) } \\
\hline \multirow{2}{*}{ Catalyst } & Cycle 1 & Cycle 2 & Cycle 3 \\
\hline $\mathrm{Pd} @ \mathbf{R}$ & 90 & 91 & 90 \\
\hline $\mathrm{Pd}\left(\mathrm{NO}_{3}\right)_{2}$ & 28 & -- & -- \\
\hline $\mathrm{Pd} @ 1$ & 69 & 63 & 67 \\
\hline
\end{tabular}




\begin{tabular}{|c|c|c|c|}
\hline \multicolumn{3}{|c|}{ Yields of conversion (\%) } \\
\hline \multirow{2}{*}{ Catalyst } & Cycle 1 & Cycle 2 & Cycle 3 \\
\hline Pd@R & 92 & 91 & 89 \\
\hline $\mathrm{Pd}(\mathrm{NO})_{2}$ & 30 & -- & -- \\
\hline $\mathrm{Pd} @ 1$ & 55 & 56 & 54 \\
\hline
\end{tabular}

Table S2. Additional Suzuki-Miyaura coupling reactions catalyzed by Pd@R catalysts

\begin{tabular}{|l|l|l|l|l|}
\hline \multicolumn{5}{|c|}{} \\
\hline entry & $\mathrm{X}$ & $\mathrm{R} 1$ & $\mathrm{R} 2$ & Conversion (\%) \\
\hline 1 & $\mathrm{Cl}$ & $\mathrm{H}$ & $\mathrm{CH}_{3}$ & 93 \\
\hline 2 & $\mathrm{Br}$ & $p-\mathrm{OCH}_{3}$ & $\mathrm{H}$ & 92 \\
\hline 3 & $\mathrm{Br}$ & $3,5-d i-\mathrm{C}_{2}\left(\mathrm{CH}_{3}\right)_{3}$ & $\mathrm{H}$ & 92 \\
\hline 4 & $\mathrm{Br}$ & $p-\mathrm{NO}_{2}$ & $\mathrm{H}$ & 89 \\
\hline 5 & $\mathrm{I}$ & $\mathrm{H}$ & $\mathrm{H}$ & 98 \\
\hline
\end{tabular}

\section{References:}

1. Delley B (2000) From molecules to solids with the $\mathrm{DMol}^{3}$ approach. J Chem Phys 113 (18):7756-7764

2. Perdew JP, Burke K, Ernzerhof M (1996) Generalized Gradient Approximation Made Simple. Phys Rev Lett 77 (18):3865-3868

3. Delley B (1990) An all-electron numerical method for solving the local density functional for polyatomic molecules. J Chem Phys 92:508-517

4. Dolg M, Wedig U, Stoll H, Preuss H (1987) Energy-adjusted abinitio pseudopotentials for the first row transition elements. J Chem Phys 86 (2):866-872

5. Bergner A, Dolg M, Küchle W, Stoll H, Preuß H (1993) Ab initio energy-adjusted pseudopotentials for elements of groups 13-17. Mol Phys 80 (6):1431-1441 